\title{
A statistical approach to the optimization of the radar ambiguity function and the chaos-based waveform design
}

\author{
Zouhair Ben Jemaa ${ }^{1}$ \\ Laboratoire RISC, ENIT, Université Tunis Elmanar, Tunis, Tunisia \\ zouhair.benjemaa@enit.rnu.tn \\ Sylvie Marcos \\ Laboratoire L2S, CNRS UMR8506, CentraleSupelec, Université Paris Sud Paris, France \\ sylvie.marcos@l2s.centralesupelec.fr \\ Safya Belghith \\ Laboratoire RISC, ENIT, Université Tunis Elmanar, Tunis, Tunisia \\ safya.belghith@enit.utm.tn
}

\begin{abstract}
In this paper we adopt a statistical approach to optimize the ambiguity function of a radar system. By considering the codes defining the transmitted waveform as realizations of a random variable we firstly show that a suitable distribution of the random variable allows to obtain good codes. Secondly we show that using the chaotic skew tent map it is possible to generate deterministic codes having the desired statistical properties. This allows to obtain an optimized global ambiguity function of the radar system. The advantage of using chaos-based sequences is that they can be easily generated in any length and number. We further improve their performance by introducing down sampling. It appears that the proposed sequences have performance quite similar to those of the sequences of the literature computationally optimized.

Keywords: Radar ambiguity function, Waveform design, Statistical approach, Chaos-based sequences, Skew tent map, Invariant probability density
\end{abstract}

\footnotetext{
${ }^{1}$ Corresponding author.
}

Preprint submitted to Signal Processing Journal

September 10, 2020 


\section{Introduction}

The primary purpose of a radar system [1] is to extract information about potentially moving targets in a given propagation environment by transmitting well-chosen waveforms and analysing the signals returned to the radar after their reflection on the targets. In particular the parameters of interest are the range and the speed of the target which can be measured by the round trip time and the Doppler frequency shift of the signal received by the radar, respectively. The system performance is based both on the receive filter and transmit waveform. We here focus on waveform design. The ambiguity function

$10[2,3]$ describes the response of a matched filter to a signal for different time delays and Doppler frequencies. The search for transmitted waveforms leading to the "best possible" radar ambiguity function, especially with regard to the low side lobes, has already been addressed in the literature [1]. In $[5,6]$ the authors presented the problem of waveform design as the nonlinear optimization of the radar ambiguity function and proposed algorithms which are relatively expensive in computation and / or complex to implement.

Waveform optimization has also recently sparked renewed interest in the context of MIMO radar [7] which consists of multiple antenna elements transmitting different waveforms toward different angles. In this context some works of the literature are inspired by the field of multi-user communications $[8,9]$, others aim to synthesize sequences by optimizing certain criteria concerning their autoand cross-correlation functions [10-14]. It however appears that the waveforms generated by the existing methods have drawbacks. Either they are limited in length, or they require important calculations, especially when we need a large 25 number of them or we want to add one.

Because the optimization of the global ambiguity function, that is to say over all time delays and Doppler frequencies, is quite difficult, other works [15-17] suggested locally optimizing the ambiguity function over a given range of time delays and Doppler frequencies. Our approach proposed here considers the global ambiguity function. 
In this paper, we propose to consider chaotic sequences as an alternative to other sequences in the literature for the design of radar waveforms. Earlier articles in the context of multi-user CDMA communication have already shown the interest of using codes based on chaos over more traditional codes $[18,19]$. Some other works have also suggested the use of chaotic sequences as candidates for the design of radar waveforms (see [20-22] and the references inside). In this paper we propose a statistical approach to exploit the features of chaos in radar system. By considering each code as a realization of a random variable we show that we can build good codes if the distribution of the random variable is

40 suitably chosen. We then consider sequences generated by the skew tent map and we show that for some values of the bifurcation parameter the invariant probability density coincides with the desired one. Note that this approach is valid for all the values of the Doppler frequency and thus allows the optimization of the global ambiguity function.

45 After briefly introducing in section 2 the ambiguity function and extracting the function of interest to be optimized, we will study in section 3 the statistical properties of the latter. In section 4 , we will show how sequences generated by the skew tent map meet the statistical properties established in the previous section and required for a good ambiguity function. We will also propose a downsampling of our sequences in order to further improve their behaviour which will be compared to computationally optimized sequences of the literature. Finally the conclusion will summary the contribution of the paper and will present future extensions.

\section{Short reminder of the radar ambiguity function}

The ambiguity function (AF) of a radar system consists of the $2 \mathrm{D}$ output, for a given time delay $\tau$ and a given Doppler frequency $\nu$, of the filter matched to the transmitted signal $s(t)$ and can be written as [7]:

$$
A(\tau, \nu)=\int s(t) s^{*}(t+\tau) e^{j 2 \pi \nu t} d t
$$


where the waveform $s(t)$ is given by:

$$
s(t)=\sum_{p=1}^{N_{c}} w_{p} u\left(t-(p-1) T_{c}\right)
$$

$N_{c}$ is the length of the sequences $\left\{w_{p}\right\}_{p=1, N_{c}}$ and $u(t)$ is a shaping function of duration $T_{c}$. (1) then becomes:

$$
A(\tau, \nu)=\sum_{p=1}^{N_{c}} \sum_{l=1}^{N_{c}} w_{p} w_{l}^{*} \widetilde{\gamma}_{p, l}^{u}(\tau, \nu)
$$

where

$$
\widetilde{\gamma}_{p, l}^{u}(\tau, \nu)=\int u\left(t-(p-1) T_{c}\right) u\left(t-(l-1) T_{c}+\tau\right) e^{j 2 \pi \nu t} d t
$$

After some calculations the ambiguity function for $\tau=k T_{c}$ then becomes:

$$
A\left(k T_{c}, \nu\right)=R_{w}(\nu, k) \alpha(\nu)
$$

where

$$
\alpha(\nu)=\int_{0}^{T_{c}}|u(t)|^{2} e^{j 2 \pi \nu t} d t
$$

and

$$
R_{w}(k, \nu)=\sum_{p=1}^{N_{c}-k} \omega_{p}^{*} \omega_{p+k} e^{-j \pi \nu(p-1) T_{c}}
$$

65 Note that in the case where $u(t)$ is the rectangular function of support $\left[0, T_{c}\right]$,

$$
\alpha(\nu)=e^{j \pi \nu T_{c}} \frac{\sin \pi \nu T_{c}}{\pi \nu}
$$

In practice, the Doppler frequency $\nu$ is usually much smaller than the bandwidth of the probing waveform so that we can safely suppose that $\left|\frac{\sin \pi \nu T_{c}}{\pi \nu}\right| \simeq T_{c}$. It then appears that the optimization of the ambiguity function reduces to the optimization of $\left|R_{w}(k, \nu)\right|$, it is to say that $\left|R_{w}(k, \nu)\right|$ for $k \neq 0$ must be the 70 smallest as possible compared to $\left|R_{w}(0,0)\right|, \forall \nu$.

We here consider phased codes so that $\omega_{p}=e^{j \pi x_{p}}$, where $x_{p}$ is a sequence in the interval $[-1,1]$. The modulus of the expression (7) (we omit the subscript $w$ in the following) becomes

$$
|R(k, \nu)|=\left|\sum_{p=1}^{N_{c}-k} e^{j \pi z_{p}(k)}\right|
$$


where

$$
z_{p}(k)=x_{p+k}-x_{p}-\nu(p-1) T_{c}
$$

75 If the sequence $x_{p}$ is randomly generated, $z_{p}(k)$ is also a random variable for every fixed integer $k \neq 0$ and Doppler frequency $\nu$. Note that for $k=0$, $|R(0, \nu)|=\left|\frac{\sin \left(\pi \nu T_{c} N_{c} / 2\right)}{\sin \left(\pi \nu T_{c} / 2\right)}\right|$ so that $|R(0,0)|=N_{c}$.

In the section below we will first analyse the statistics of the modulus $R=$

so $\left|Z_{n}\right|$, for a fixed $n$, with

$$
Z_{n}=\sum_{p=1}^{n} e^{j \pi y_{p}}
$$

where $n$ is large enough and $y_{p}$ is a random variable. We will first establish the desired statistical properties for the random variable $R=\left|Z_{n}\right|$, in order to minimize its mean and variance which are related to the side lobes of the ambiguity function. We will then consider different distributions for $y_{p}$ in (11).

${ }_{85}$ The optimisation of $R=\left|Z_{n}\right|$ through the statistics of $y_{p}$ will then be useful to the choice of the sequences $x_{p}$ allowing the desired properties of $z_{p}$ in (9) and 10 .

We will also analyse the influence of the deterministic term in 10 corresponding to the Doppler frequency $\nu$.

\section{3. Statistical properties of $R=\left|Z_{n}\right|$}

Let $y_{p}$ be an i.i.d random variable. By noting $\alpha$ and $\beta$ the mean values of the real and imaginary parts of $Z_{n}$, and by $s_{1}$ and $s_{2}$ their variances respectively we introduce the following notations as in 23 .

$$
\left\{\begin{array}{l}
X=\frac{R}{\sqrt{s_{1}+s_{2}}} \\
B=\frac{\alpha}{\sqrt{s_{1}+s_{2}}} \\
K=\sqrt{\frac{s_{2}}{s_{1}}}
\end{array}\right.
$$

In the case of symmetric distribution with respect to 0 (i.e. $\beta=0$ ) the distribution of the random variable $X$ is 23 .

$$
f_{X}(x)=\frac{K^{2}+1}{K} x \exp \left[-\frac{K^{2}+1}{2}\left(B^{2}+\frac{K^{2}+1}{2 K^{2}} x^{2}\right)\right]
$$




$$
\sum_{m=0}^{\infty}(-1)^{m} \varepsilon_{m} I_{m}\left(\frac{K^{4}-1}{4 K^{2}} x^{2}\right) I_{2 m}\left[B\left(1+K^{2}\right) x\right]
$$

where

$$
\varepsilon_{m}= \begin{cases}1, & \text { if } m=0 \\ 2, & \text { if } m \neq 0\end{cases}
$$

$I_{m}$ is the modified Bessel function of the first kind.

In 23 it has been shown that:

- by varying $K$ the distribution is only slightly modified, so we will consider $K=1$ in the following.

- the mean and the variance are the smallest for $B=0$, corresponding to $\alpha=0$.

The distribution $(13)$ is then almost optimal in the sense that for a given value $x_{0}$ of $X$ the probability $P\left(X<x_{0}\right)$ is the greatest for $B=0$. This is illustrated in Figure 1 for $K=1,2$ and $B=0,0.5,1,2$.

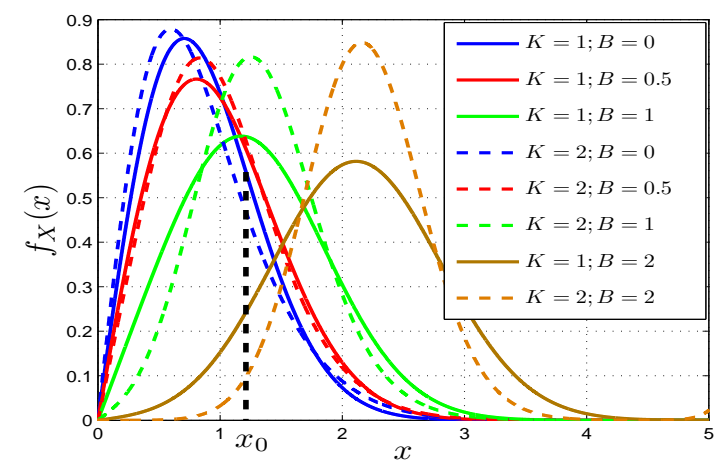

Figure 1: Distribution $f_{X}(x)$ for different values of $K$ and $B$

105

For $K=1$ the distribution $(13)$ is then reduced to

$$
f_{X}(x)=2 x e^{-\left(B^{2}+x^{2}\right)} I_{0}(2 B x)
$$

Using (12) we retrieve the distribution of the random variable $R=\left|Z_{n}\right|$

$$
f_{R}(r)=2 \frac{r}{s_{1}+s_{2}} e^{-\left(B^{2}+\frac{r^{2}}{s_{1}+s_{2}}\right)} I_{0}\left(2 B \frac{r}{\sqrt{s_{1}+s_{2}}}\right)
$$


- For $\alpha \neq 0$ and thus $B \neq 0$ the distribution 15 is the Rice distribution

- For $\alpha=0$ and thus $B=0$ we obtain the Rayleigh distribution

$$
f_{R}(r)=2 \frac{r}{s_{1}+s_{2}} e^{-\frac{r^{2}}{s_{1}+s_{2}}}
$$

110

The mean value and the variance of the distribution $(16)$ are:

$$
\left\{\begin{array}{l}
E_{R}=\frac{\sqrt{\left(s_{1}+s_{2}\right) \pi}}{2} \\
V_{R}=\left(1-\frac{\pi}{4}\right)\left(s_{1}+s_{2}\right)
\end{array}\right.
$$

This distribution will then be considered as a reference, i.e. as said above, a sequence $y_{p}$ in 11 yielding to such a Rayleigh distribution will be considered as optimal.

In the following we will consider several distributions for $y_{p}$. First note that since $y_{p}$ is supposed to follow an i.i.d distribution we have

$$
\alpha=n E\left[\cos \pi y_{p}\right]
$$

\subsection{Case $y_{p}$ uniformly distributed in $[-a, a] ; a \leq 1$}

In this case

$$
\beta=0 ; \quad \alpha=n \operatorname{sinc}(a)
$$

where $\operatorname{sinc}(x)=\frac{\sin (\pi x)}{\pi x}$.

$$
\begin{gathered}
s_{1}=\frac{n}{2}\left(1+\operatorname{sinc}(2 a)-2 \operatorname{sinc}^{2}(a)\right) \\
s_{2}=\frac{n}{2}(1-\operatorname{sinc}(2 a))
\end{gathered}
$$

which gives

$$
\left\{\begin{array}{l}
s_{1}+s_{2}=n\left(1-\operatorname{sinc}^{2}(a)\right) \\
B=\sqrt{n} \frac{\operatorname{sinc}(a)}{\sqrt{1-\operatorname{sinc} c^{2}(a)}}
\end{array}\right.
$$

The Rayleigh distribution (16) is obtained for $a$ an integer. Indeed in this case $\operatorname{sinc}(a)=0$ which gives $B=0, K=1$ and $s_{1}+s_{2}=n$. Figure 2 exhibits an example of $f_{R}(r)$ for $n=1023$ and for different values of $a$. In the case of the Rayleigh distribution (16) and from (17) the mean value and variance of ${ }_{125} R=\left|Z_{n}\right|$ are 


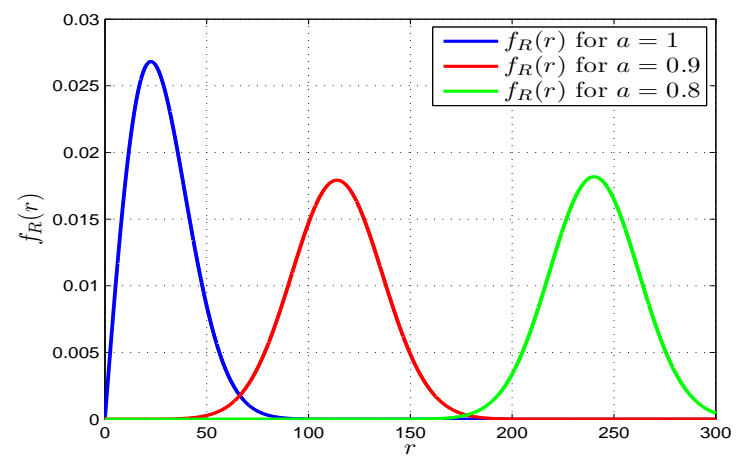

Figure 2: The distribution $f_{R}(r)$ for $n=1023$ and $a=1 ; 0.9 ; 0.8$

$$
\left\{\begin{array}{l}
E_{R}=\frac{\sqrt{n \pi}}{2} \\
V_{R}=\left(1-\frac{\pi}{4}\right) n
\end{array}\right.
$$

Note that, in practice, these mean and variance values should be compared to the maximum peak of the ambiguity function, i.e. $|R(0,0)|=n$, and its square value, respectively. Thus we considered the two more significant features: the normalized mean $E_{n}=\frac{E_{R}}{n}$ and variance $V_{n}=\frac{V_{R}}{n^{2}}$, we obtain

$$
\left\{\begin{array}{l}
E_{n}=\frac{1}{2} \sqrt{\frac{\pi}{n}} \\
V_{n}=\left(1-\frac{\pi}{4}\right) \frac{1}{n}
\end{array}\right.
$$

It then appears that the mean side lobe to maximal peak ratio of the ambiguity function vanishes as $n$ increases as illustrated in Figure 3 .

\subsection{Case $y_{p}$ uniformly distributed in $[-a, a] ; a>1$}

Due to the fact that $e^{j \pi y_{p}}$ is 2 -periodic, a random variable $\tilde{y}_{p}$ in $[-1,1]$ satisfying $e^{j \pi y_{p}}=e^{j \pi \tilde{y}_{p}}$ corresponds to the random variable $y_{p}$, see the example plotted in red line on Figure 4 . Consequently, the statistical properties of $\left|Z_{n}\right|$ are the same for $y_{p}$ and $\tilde{y}_{p}$. It is easy to show that $\tilde{y}_{p}$ is given by:

$$
\tilde{y}_{p}=\left\{\begin{array}{lll}
y_{p}-\left\lfloor y_{p}\right\rfloor-1 & \text { if } & \left\lfloor y_{p}\right\rfloor \text { is odd } \\
y_{p}-\left\lfloor y_{p}\right\rfloor & \text { if } & \left\lfloor y_{p}\right\rfloor \text { is even }
\end{array}\right.
$$



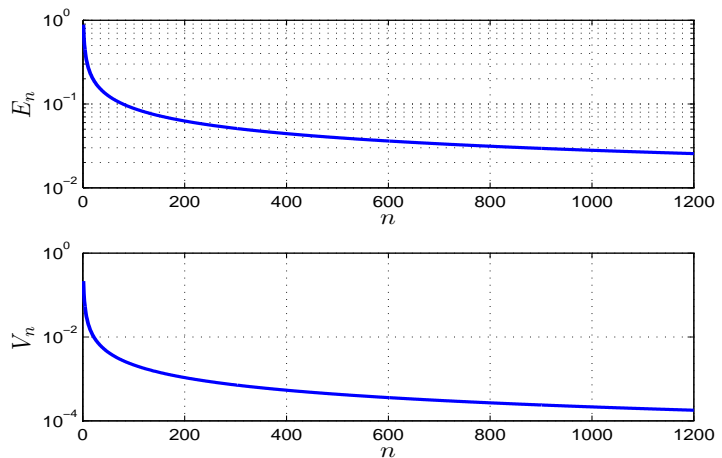

Figure 3: Mean and variance of the Rayleigh distribution versus sequence length $n$

$\lfloor x\rfloor$ is the floor of $x$. Let $y_{p}$ be uniformly distributed in $[-a, a]$ where $a>1$. We show in the appendix that $f_{\tilde{y}_{p}(y)}$ has two expressions according to $\lfloor a\rfloor$.

- if $\lfloor a\rfloor$ is even, $\lfloor a\rfloor=2 l$

$$
f_{\tilde{y}_{p}(y)}=\left\{\begin{array}{lll}
\frac{l}{a} & \text { if } & a-2 l \leq|y| \leq 1 \\
\frac{2 l+1}{2 a} & \text { if } & 0 \leq|y| \leq a-2 l
\end{array}\right.
$$

- if $\lfloor a\rfloor$ is odd, $\lfloor a\rfloor=2 l+1$

$$
f_{\tilde{y}_{p}(y)}=\left\{\begin{array}{lll}
\frac{2 l+1}{2 a} & \text { if } & 0 \leq|y| \leq-a+2 l+2 \\
\frac{l+1}{a} & \text { if } & -a+2 l+2 \leq|y| \leq 1
\end{array}\right.
$$

In Figure 4, we plotted the probability density of $f_{y}(y)$ and $f_{\tilde{y}}(y)$ in the two cases $\lfloor a\rfloor$ odd $(\lfloor a\rfloor=5)$ and $\lfloor a\rfloor$ even $(\lfloor a\rfloor=6)$. By an easy computation of $\alpha=\int_{-1}^{1} \cos (\pi y) f_{\tilde{y}}(y) d y$ we found that

$$
\alpha= \begin{cases}-\frac{\sin (\pi(2 l+2-a))}{2 \pi a} & \text { if }\lfloor a\rfloor=2 l+1 \\ \frac{\sin (\pi(a-2 l))}{2 \pi a} & \text { if }\lfloor a\rfloor=2 l\end{cases}
$$

In both cases $2 l+2-a$ and $a-2 l \in[0,1[, \alpha=0$ if and only if $a$ is an integer corresponding to the uniform distribution in $[-1,1]$. In other words we obtain $R$ with the Rayleigh distribution (16) if and only if $\alpha=B=0$. 

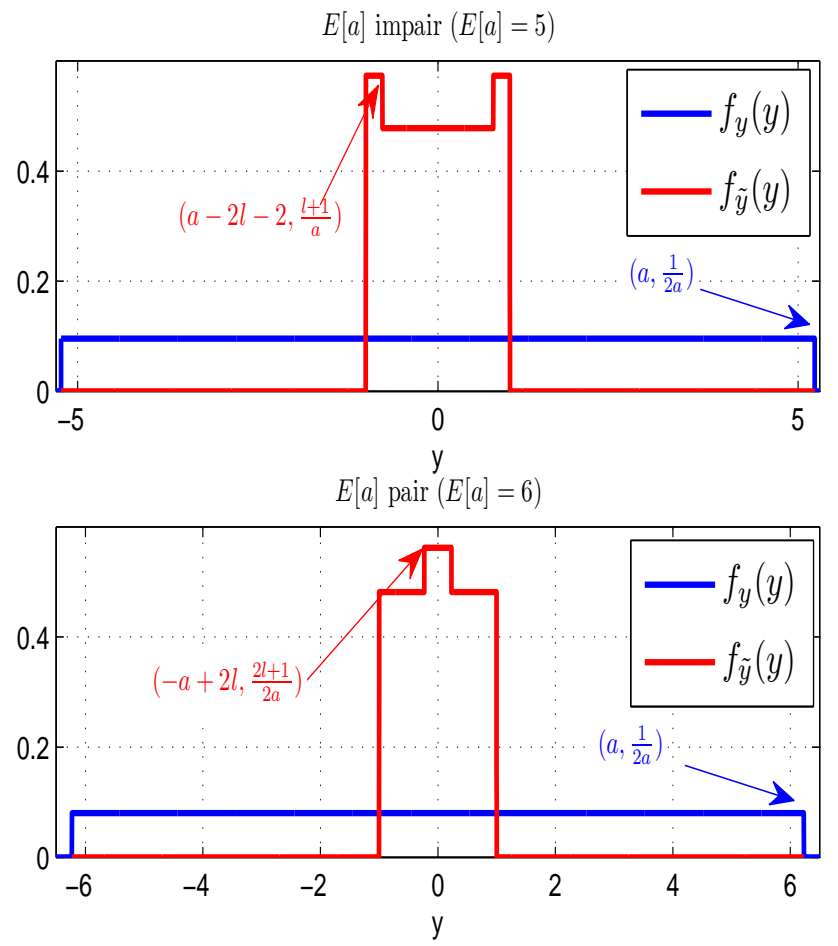

Figure 4: $f_{y}(y)$ and $f_{\tilde{y}}(y)$ in the case of uniform distribution.

\subsection{Case of $y_{p}$ with a triangular distribution in $[-a, a]$}

First note that this distribution is obtained when $y_{p}=z_{p}(k)$ in 10 with $\nu=0$ and uniformly distributed $x_{p}$ and $x_{p+k}$.

150 We show in the appendix that $f_{\tilde{y}}(y)$ can have two expressions according to $\lfloor a\rfloor$ :

- if $\lfloor a\rfloor$ is even, $\lfloor a\rfloor=2 l$

$$
f_{\tilde{y}}(y)=\left\{\begin{array}{lll}
\frac{(2 l+1) a-2 l(l+1)-|y|}{a^{2}} & \text { if } \quad 0 \leq|y| \leq a-2 l \\
\frac{2 l a-2 l^{2}}{a^{2}} & \text { if } \quad a-2 l \leq|y| \leq 1
\end{array}\right.
$$

- if $\lfloor a\rfloor$ is odd, $\lfloor a\rfloor=2 l+1$

$$
f_{\tilde{y}}(y)= \begin{cases}\frac{(2 l+1) a-2 l(l+1)-|y|}{a^{2}} & \text { if } \quad 0 \leq|y| \leq 2 l+2-a \\ \frac{2(l+1) a-2(l+1)^{2}}{a^{2}} & \text { if } \quad 2 l+2-a \leq|y| \leq 1\end{cases}
$$



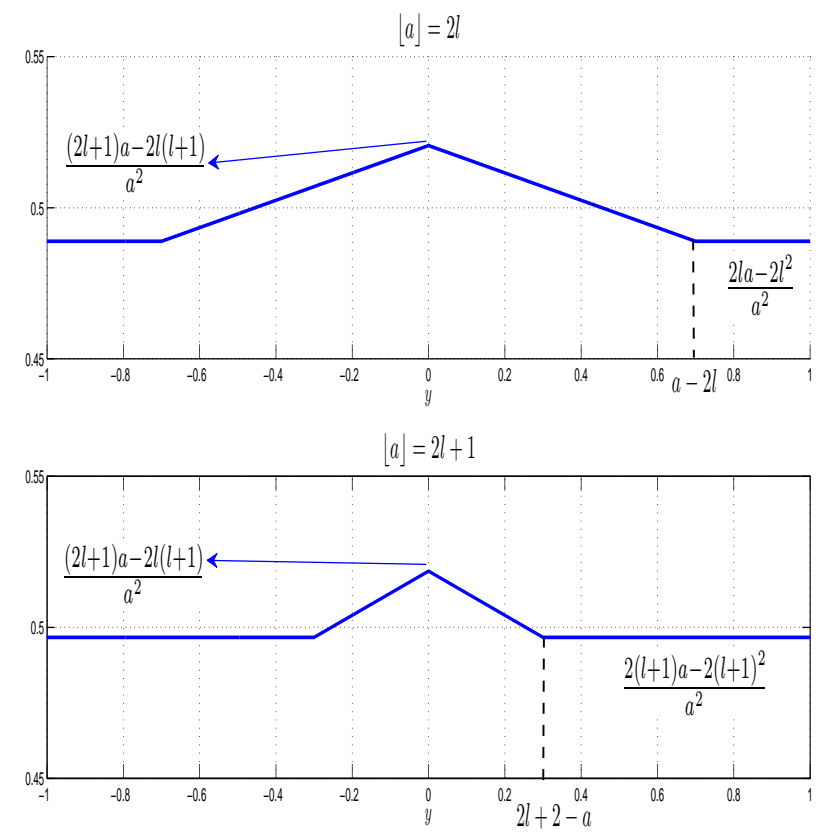

Figure 5: $f_{\tilde{y}}(y)$ when $y_{p}$ has a triangular distribution in [-a,a] in the cases $a$ even $(a=2 l)$ and $a$ odd $(a=2 l+1)$

In Figure 5 we plotted this distribution in both cases. Note that:

- for $\lfloor a\rfloor$ even, when $a$ is an integer, i.e. $a=2 l$ we obtain the uniform distribution in $[-1,1]$.

- for $\lfloor a\rfloor$ odd, when $a$ is an integer, i.e. $a=2 l+1$ we obtain the triangular distribution in $[-1,1]$.

These two results explain the expressions of $\alpha$ obtained by the computation of $\alpha=\int_{-1}^{1} \cos (\pi y) f_{\tilde{y}}(y) d y$ and given below

$$
\alpha= \begin{cases}-2 \frac{1-\cos (\pi(a-2 l))}{\pi^{2} a^{2}} & \text { if }\lfloor a\rfloor=2 l \\ 2 \frac{1-\cos (\pi(2 l+2-a))}{\pi^{2} a^{2}} & \text { if }\lfloor a\rfloor=2 l+1\end{cases}
$$

160 When $a=2 l$ we obtain $\alpha=0$ giving the Rayleigh distribution $(16)$, when $a=2 l+1$ the expression 29 is maximal and equal to $\frac{4}{\pi^{2} a^{2}}$ corresponding to a 
Rice distribution 15.

\subsection{Case of $y_{p}$ centered and gaussian distributed}

This distribution is obtained when $x_{p}$ and $x_{p+k}$ in (10) are independent gaussian and centred variables and $\nu=0$. When $f_{y}(y)$ is gaussian, $f_{\tilde{y}}(y)$ has

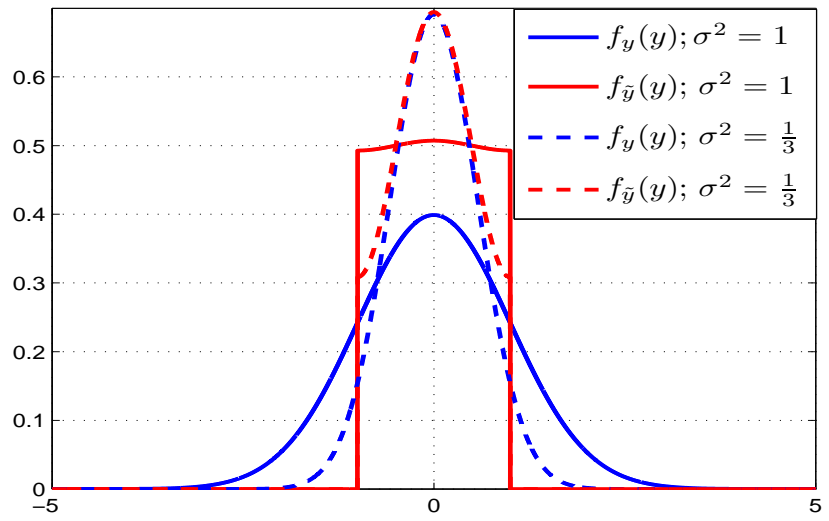

Figure 6: $f_{y}(y)$ et $f_{\tilde{y}(y)}$ in the case of centered and gaussian distribution

165

one of the two shapes represented in Figure 6, according to the variance $\sigma^{2}$ :

- $\sigma<1$ we obtain a density that differs very much from the uniform one, (as for $\sigma^{2}=\frac{1}{3}$ in the figure).

- $\sigma \geq 1$, the distribution $f_{\tilde{y}}(y)$ is almost uniform in $[-1,1]$.

\subsection{Histograms of $\left|Z_{n}\right|$}

To confirm the results of the discussion above we plotted in Figure 7 the histograms of $\left|Z_{n}\right|$ for the previously considered distributions with the Rayleigh distribution in red line. These histograms are computed for the case $n=1023$ using 10000 realizations of $y_{p}$. We obtain a Rayleigh distribution of $R=\left|Z_{n}\right|$ when $y_{p}$ follows the following distributions:

- uniform distribution in $[-q, q], q$ an integer; 
- triangular distribution in $[-q, q], q \geq 2$ an even integer;

- centered and gaussian distribution with variance greater or equal to 1 ;

For these results we can see that the average and the variance are very close $E_{n} \cong 28.3454, V_{n} \cong 219.5377$. In the other cases, we obtain a distribution which is very different from the Rayleigh distribution.
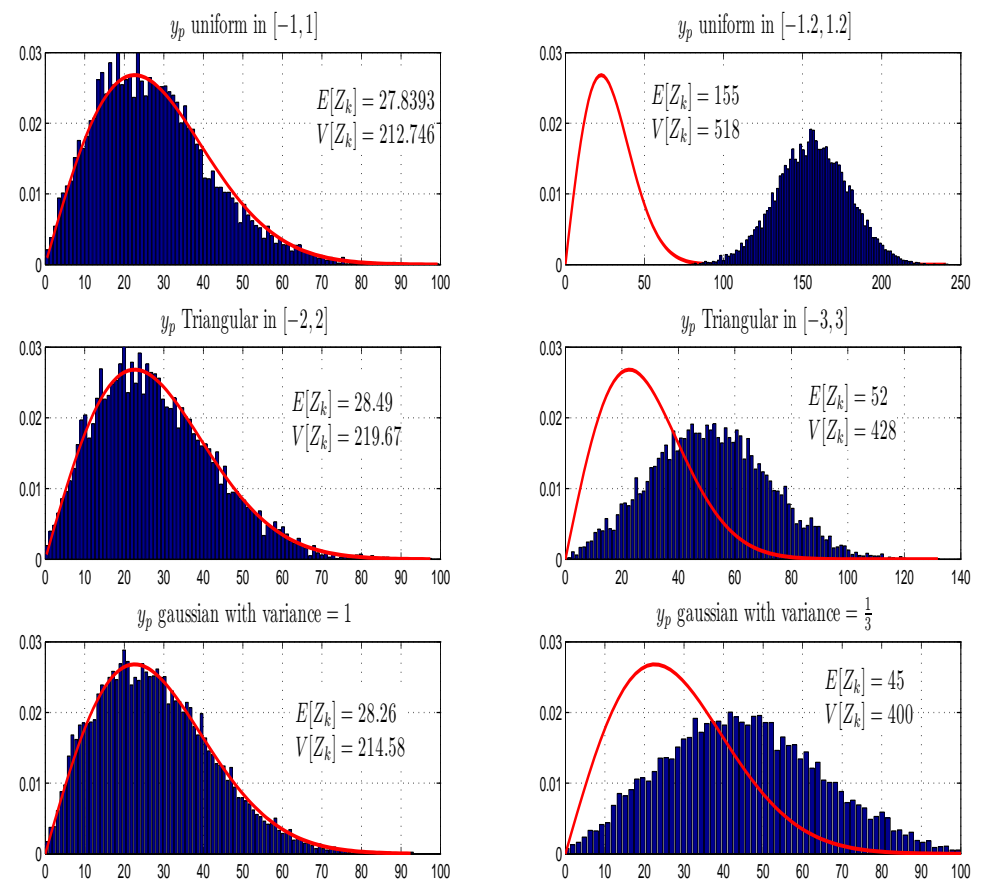

Figure 7: Histograms of $Z_{k}$ for different distribution of $y_{p}$

3.6. Statistics of $R=\left|Z_{n}\right|$ in the case $y_{p}=u_{p}+\nu(p-1) T_{c}$

In this section we tackle the influence of the presence of the determinisic term $\nu(p-1) T_{c}$ on the statistics of $R$. We consider $y_{p}=u_{p}+\nu(p-1) T_{c}$ where $u_{p}$ are i.i.d. random variables. It is easy to see that as the $u_{p}$ are i.i.d. $E\left[e^{j \pi u_{p}}\right]$ 
is a constant (not depending on $p$ ) denoted by $A$ and

$$
E\left[Z_{n}\right]=A \sum_{p=1}^{n} e^{j \pi(p-1) \nu T_{c}}
$$

In the case $\nu T_{c}=2 q$ where $q$ is an integer, $E\left[Z_{n}\right]=A n$. If $A=0$ the real part of $Z_{n}$ is also zero and consequently $\alpha=0$ and $B=0$ which are the conditions so that $R=\left|Z_{n}\right|$ has a Rayleigh type distribution (see [23]).

In case $\nu T_{c} \neq 2 q$ :

$$
E\left[Z_{n}\right]=A \frac{e^{j \pi n \nu T_{c} / 2}}{e^{j \pi \nu T_{c} / 2}} \frac{\sin \left[\pi n \nu T_{c} / 2\right]}{\sin \left[\pi \nu T_{c} / 2\right]}
$$

Since $\left|\sin \left[\pi n \nu T_{c} / 2\right]\right| \leq n\left|\sin \left[\pi \nu T_{c} / 2\right]\right|$, we obtain

$$
\left|\operatorname{Real}\left(E\left[Z_{n}\right]\right)\right| \leq\left|E\left[Z_{n}\right]\right| \leq|A| n
$$

Also in this case if $A=0$, the real part of $E\left[Z_{n}\right]$ is zero, thus $\alpha=0$ and $B=0$ which are the conditions so that $R=\left|Z_{n}\right|$ has a Rayleigh type distribution.

To illustrate this idea we plotted on Figure 8 the histograms of $\left|Z_{n}\right|$ for $y_{p}$ uniform in $\mid-1.2,1.2]$. We can see that the distribution of $\left|Z_{n}\right|$ differs from the desired Rayleigh one only when $\nu=0$. This could be explained by saying that the presence of the deterministic term allows uniform scattering of the points on the unit circle when the distribution of $u_{p}$ is not uniformly distribution in $200[-1,1]$.

Thus the presence of the deterministic term $\nu(p-1) T_{c} ; \nu \neq 0$ makes every sequence a good one since it allows scattering the points on the unit circle. We can conclude that the important thing is to optimize $\left|Z_{n}\right|$ for $\nu=0$.

\section{Ambiguity function for chaotic sequences}

In this section we consider chaotic sequences $\left\{x_{p}\right\}$ generated by $x_{p+1}=$ $T_{\mu}\left(x_{p}\right)$ and an initial condition $x_{0} \in[-1,1]$ where $\mu \in[-1,1] ; T_{\mu}(x)$ is the piece-wise linear skew tent map defined in $[-1,1]$ by

$$
T_{\mu}(x)=\left\{\begin{array}{lrr}
\frac{2}{\mu-1} x-\frac{1+\mu}{\mu-1} & \text { if } & \mu<x \leq 1 \\
\frac{2}{\mu+1} x-\frac{\mu-1}{\mu+1} & & \text { otherwise }
\end{array}\right.
$$



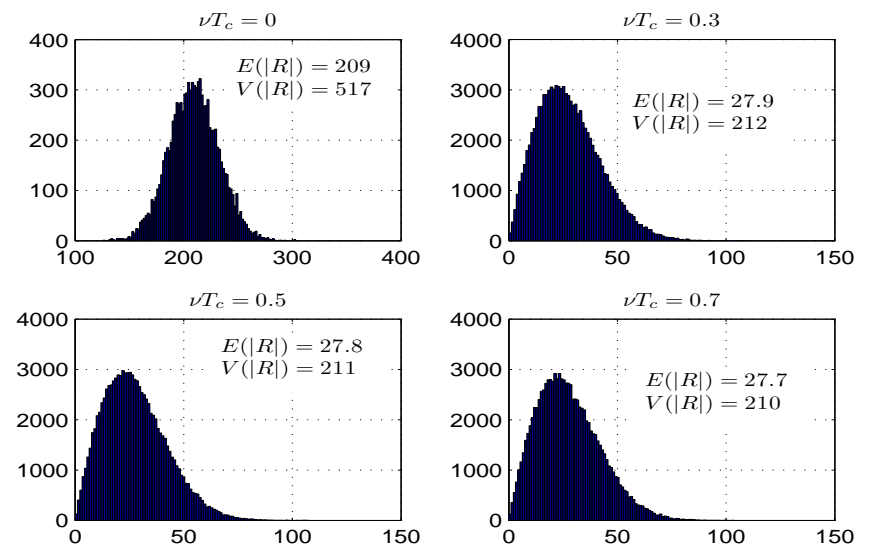

Figure 8: Histograms of $\left|Z_{k}\right|$ in the case of $y_{p}$ uniform in $[-1.2,1.2]$

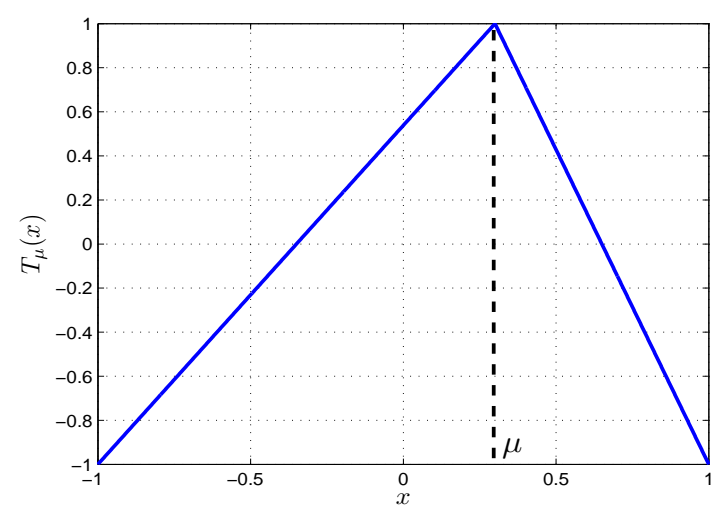

Figure 9: Curve of $T_{\mu}(x)$ 
The curve of $T_{\mu}(x)$ is given on Figure 9. The invariant probability density of the variable $x_{p}$ is the uniform distribution in the interval $[-1,1]$ [24] i.e. for $N$ large enough $x_{p}, p=0,1, \ldots, N$ could be considered as a realization of a random variable uniformly distributed in $[-1,1]$. The idea is to use such sequences in the radar system described above with $y_{p}=g_{k}\left(x_{p}\right)$ for a given integer $k$ where

$$
g_{k}(x)=T_{\mu}^{k}(x)-x
$$

To analyze the statistics of $y_{p}$ for a given $k$, we plotted the curve of $y=g_{k}(x)$ in Figure 4 for $k=4$ (red line). The argument and the value of the maxi-

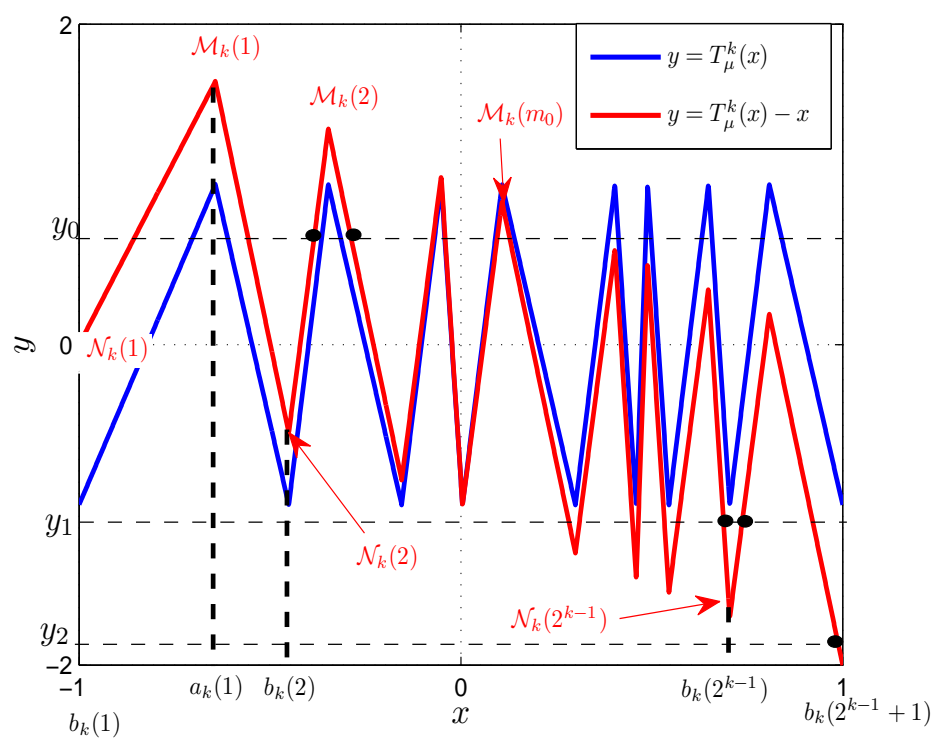

Figure 10: Curves of $y=g_{k}(x) ; k=4$

215$$
\left(b_{k}(m), \mathcal{N}_{k}(m)\right), 1 \leq m \leq 2^{k-1}+1 \text { where }
$$$$
\mathcal{M}_{k}(m)=1-a_{k}(m), 1 \leq m \leq 2^{k-1}
$$$$
\mathcal{N}_{k}(m)=-1-b_{k}(m), 1 \leq m \leq 2^{k-1}+1
$$ 
$a_{k}(m)$ and $b_{k}(m)$ are defined by the following recursive relations [25]:

220

$$
\begin{gathered}
a_{1}(1)=\mu, \quad b_{1}(1)=-1, \quad b_{1}(2)=1 \\
a_{k+1}(2 m-1)=\frac{\mu+1}{2}\left[a_{k}(m)-b_{k}(m)\right]+b_{k}(m)
\end{gathered}
$$

$$
\begin{gathered}
a_{k+1}(2 m)=\frac{\mu+1}{2}\left[a_{k}(m)-b_{k}(m+1)\right]+b_{k}(m+1) \\
b_{k+1}(2 m-1)=b_{k}(m) \\
b_{k+1}(2 m)=a_{k}(m) \\
b_{k+1}(2 m+1)=b_{k}(m+1)
\end{gathered}
$$

The curve of $T_{\mu}^{k}(x)-x$ consists of alternately $2^{k-1}$ increasing and decreasing

225

230

$$
\begin{aligned}
C_{k}(m) & =\frac{a_{k}(m)-b_{k}(m+1)}{2-a_{k}(m)+b_{k}(m+1)} \\
D_{k}(m) & =\frac{a_{k}(m)+b_{k}(m+1)}{2-a_{k}(m)+b_{k}(m+1)}
\end{aligned}
$$

- in the $m^{\text {th }}$ decreasing line we have

$$
x=C_{k}(m) y+D_{k}(m)
$$

Let $X$ be a random variable following the uniform distribution in $[-1,1]$ and $Y=T_{\mu}^{k}(X)-X$. The probability $\left.P(Y<y)=P\left(X \in g_{k}^{-1}(]-2, y\right]\right)$. Since $X$ is supposed to be uniformly distributed in $[-1,1]$ we can confirm the following 235 points about the distribution of $Y$.

- if $y \leq-2$ the probability $P(Y<y)=0$. 
- if $y \geq \mathcal{M}_{k}(1)$ the probability $P(Y<y)=1$.

- if $0 \leq y_{0} \leq \mathcal{M}_{k}(1)$ as shown in the figure there exists an unique integer $m_{0}, 1 \leq m_{0} \leq 2^{k-1}-1$ such that $y_{0} \in\left[\mathcal{M}_{k}\left(m_{0}+1\right), \mathcal{M}_{k}\left(m_{0}\right)\right]$. Using (37) and (38) we can show easily that

$$
P\left(Y<y_{0}\right)=\frac{1}{2}\left(2-\sum_{m=1}^{m_{0}}\left[C_{k}(m)-A_{k}(m)\right] y_{0}+\left[D_{k}(m)-B_{k}(m)\right]\right)
$$

- if $\mathcal{N}_{k}\left(2^{k-1}\right) \leq y_{1} \leq 0$ there exists an integer $m_{1}, 1 \leq m_{1} \leq 2^{k-1}-1$ such that $y_{1} \in\left[\mathcal{N}_{k}\left(m_{1}+1\right), \mathcal{N}_{k}\left(m_{1}\right)\right]$. In this case

$$
\begin{aligned}
P\left(Y<y_{1}\right)= & \frac{1}{2} \sum_{m=1}^{m_{1}}\left[\left(A_{k}(m)-C_{k}(m)\right) y_{1}+B_{k}(m)-D_{k}(m)\right] \\
& +\frac{1}{2}\left[2-\left(C_{k}\left(2^{k-1}\right) y_{1}+D_{k}\left(2^{k-1}\right)\right)\right]
\end{aligned}
$$

- $-2 \leq y_{2} \leq \mathcal{N}_{k}\left(2^{k-1}\right)$

$$
P\left(Y<y_{2}\right)=\frac{1}{2}\left(2-\left(C_{k}\left(2^{k-1}\right) y_{2}+D_{k}\left(2^{k-1}\right)\right)\right)
$$

By deriving the function $P(Y<y)$ in all the cases above we obtain the probability density $f_{\mu, k}(y)$ of $Y=T_{\mu}^{k}(X)-X$

$$
f_{\mu, k}(y)=\left\{\begin{array}{ccc}
\frac{\sum_{m=1}^{m_{y}} \gamma(k, m)}{2}, & \text { if } & 0 \leq y \leq \mathcal{M}_{k}(1) \\
\frac{\sum_{m=1}^{m_{y}} \gamma(k, m)+C_{k}\left(2^{k-1}\right)}{2}, & \text { if } & \mathcal{N}_{k}\left(2^{k-1}\right) \leq y \leq 0 \\
\frac{-C\left(k, 2_{k-1}\right)}{2} & \text { if } & -2<y \leq \mathcal{N}_{k}\left(2^{k-1}\right) \\
0 & & \text { otherwise }
\end{array}\right.
$$

where $m_{y}$ is the integer satisfying $y \in\left[\mathcal{M}_{k}\left(m_{y}+1\right), \mathcal{M}_{k}\left(m_{y}\right)\right]$ if $0 \leq y \leq 1-a_{k}(1)$ or satisfying $y \in\left[\mathcal{N}_{k}\left(m_{y}+1\right), \mathcal{N}_{k}\left(m_{y}\right)\right]$ if $-1-b_{k}\left(2^{k-1}\right) \leq y<0$, and

$$
\gamma(k, m)=A(k, m)-C(k, m)
$$

Figure 11 exhibits the probability density of $y=T_{\mu}^{k}(x)-x$ in blue line for $\mu=0.7$ and for different values of $k$. In red line we plotted the probability density of $\tilde{y}$ corresponding to $y$ as defined in the previous section. From the 

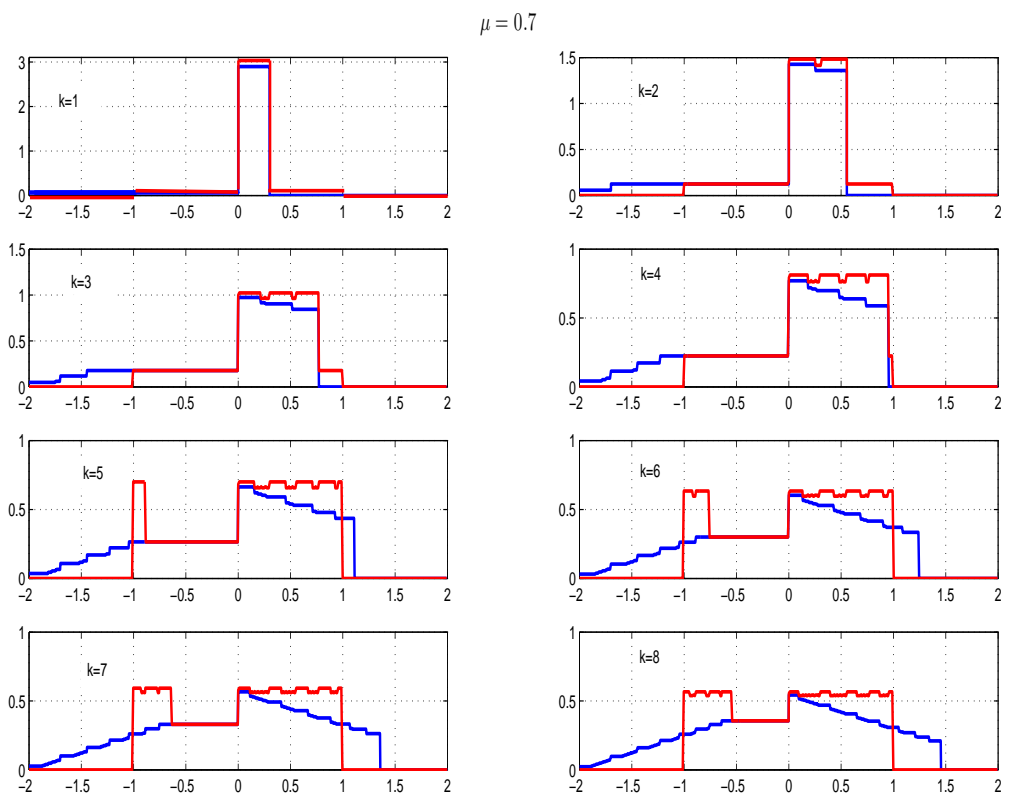

Figure 11: Probability density of $y=T_{\mu}^{k}(x)-x$ (Blue line) and $\tilde{y}$ (red line) for $\mu=0.7$ 

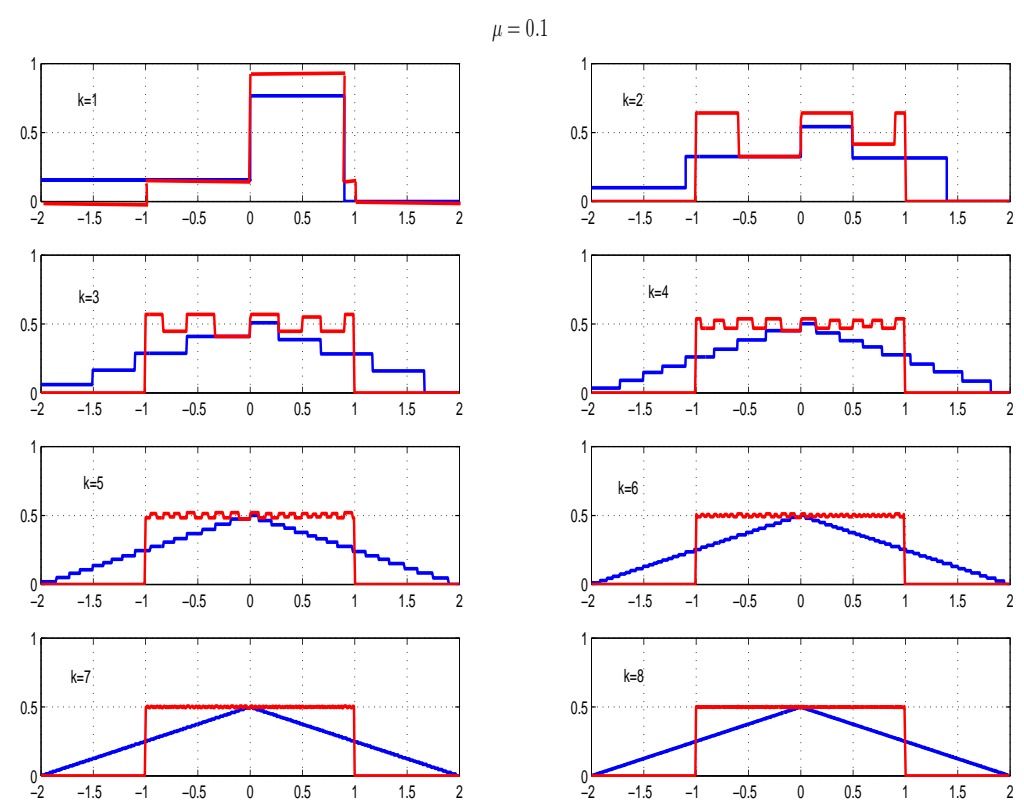

Figure 12: Probability density of $y=T_{\mu}^{k}(x)-x$ (Blue line) and $\tilde{y}$ (red line) for $\mu=0.1$ 
previous discussion $\tilde{y}$ should be uniform in the interval $[-1,1]$ in order to have a Rayleigh distribution for $R=\left|Z_{n}\right|$ and consequently to minimize the side lobes of the ambiguity function.

255 We can see that after a few iterations the probability density of $y=T_{\mu}^{k}(x)-x$ tends to the triangular distribution in the interval $[-2,2]$ and thus $\tilde{y}$ follows the uniform distribution in the interval $[-1,1]$. We did the same thing for $\mu=0.1$; the results are shown in the Figure 12. We have almost the same behaviour, with the difference that for the case $\mu=0.1$ the distribution converges more quickly with respect to the case $\mu=0.7$. In the general case we obtain a distribution that converges to the triangular one for all values of $\mu$, subsequently we have to look for parameter $\mu$ that yields the uniform distribution for the smallest value of iteration $k$.

To measure the resemblance of $f_{\mu, k}(y)$ with the uniform distribution on the interval $[-1,1]$ we considered the criteria

$$
C(\mu, k)=\int_{-1}^{1}\left(f_{\mu, k}(x)-\frac{1}{2}\right)^{2} d x
$$

We plotted on Figure $13 C(\mu, k)$ versus the iteration number $k$ and for different

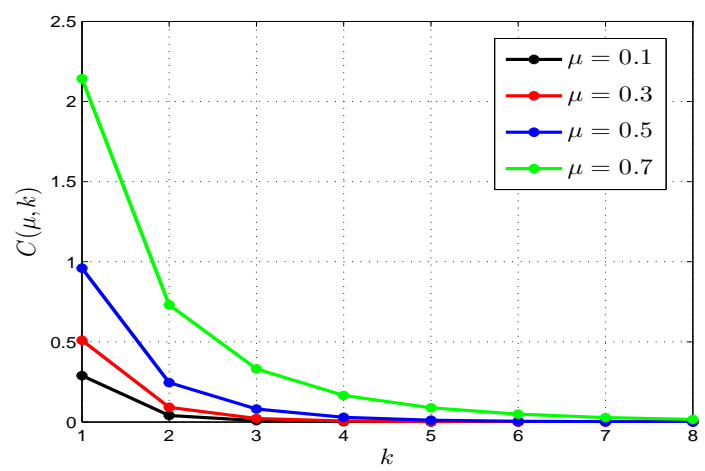

Figure 13: $C(\mu, k)$ for different values of $\mu$ and $k$

values of $\mu$. It is clear that the case $\mu=0.1$ allows the best value of $C(\mu, k)$. To see the impact of this result, we plotted on Figure 14 the main term (9) involved in the ambiguity function (5) as a function of $\nu$ and for $k=1,2,3$ and 
allows side lobes in the ambiguity function $(9)$ smaller than the case $\mu=0.7$.

We have considered here only the first values of $k$ since for higher values
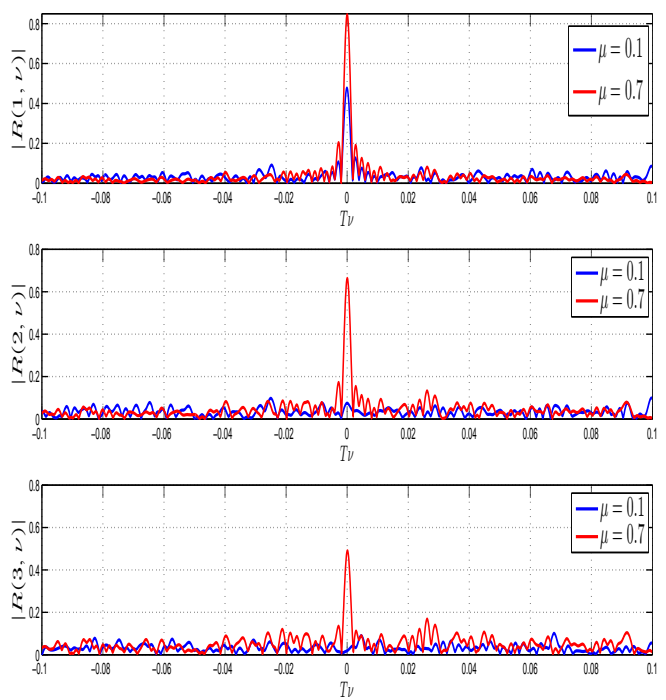

Figure 14: $|R(k, \nu)|$ for $k=1,2,3 ; \mu=0.1$ and $\mu=0.7$

$|R(k, \nu)|$ behaves similarly regardless of the bifurcation parameter of the chaotic sequence. Indeed the invariant distribution reaches the uniform distribution for large values of $k$ as explained above. In addition as previously explained for $\nu \neq 0$ the phase coded sequence is distributed on the unit circle giving good behaviour of $|R(k, \nu)|$ even with bad distribution; this explains the similarity of the peaks of $|R(k, \nu)|$ for $\mu=0.1$ and $\mu=0.7$

This can also be seen in Figures 15 and 16 where the contours of the ambiguity functions are plotted for $\mu=0.1$ and $\mu=0.7$ respectively. We zoomed in on the small values of $k$ and $\nu$ because in the other regions, the behaviour of the ambiguity function is almost the same for $\mu=0.1$ and $\mu=0.7$. In these Figures and in Figures 20 and 21 below we have removed the maximum peaks of $|R(0, \nu)|$; indeed $|R(0, \nu)|$ is independent of the phase coded sequences (as 
wn under equations $(9)-(10))$.

In Figure 17 we have plotted the maximum value of the first side lobe of the

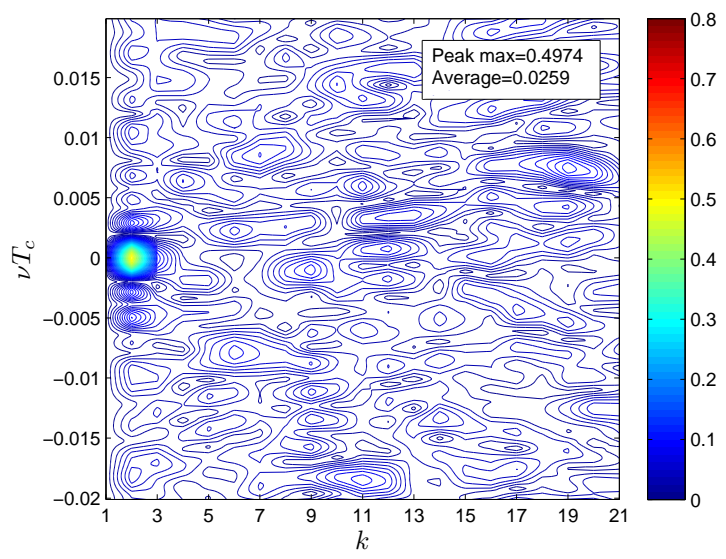

Figure 15: Ambiguity function $(|R(0, \nu)|$ removed) obtained with chaotic waveform $(\mu=0.1)$

ambiguity function and the Lyapunov exponent (the well-known parameter that characterizes the instability of a dynamic system) with respect to the bifurcation parameter $\mu$. We can see that the more chaotic the sequences, i.e. the larger the Lyapunov exponent, the lower the maximum value of the first side lobe of the ambiguity function.

It should be noted that other chaotic transformations than the skew tent map could have been envisaged in order to obtain a better distribution of the sequence generated in particular in the first iterations and could thus avoid the high peaks around zero (that is to say for $k=1$ ) in the ambiguity function. If we have considered here the skew tent map as an example for generating chaotic sequences, it is because in our opinion, its theoretical analysis is easier than that of other chaotic transformations. In the next section, we propose a way to construct sequences extracted from the sequences generated by the skew 


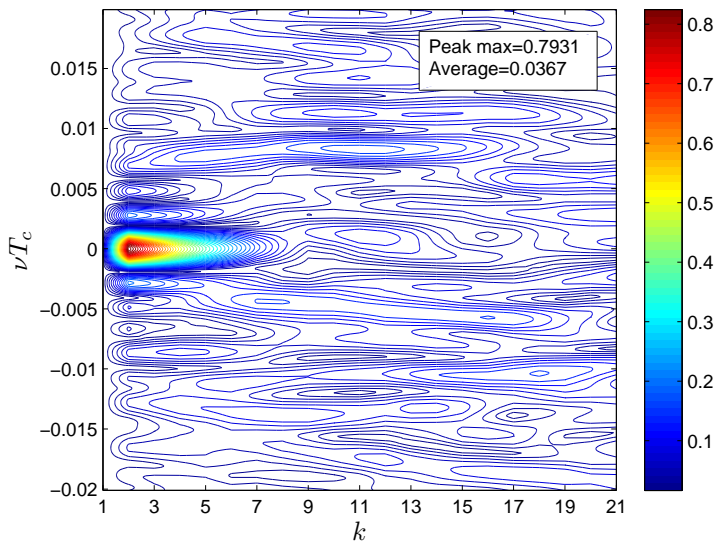

Figure 16: Ambiguity function $(|R(0, \nu)|$ removed) obtained with chaotic waveform $(\mu=0.7)$

\section{Ambiguity function for down-sampled chaotic sequences}

We have shown in the previous section how a chaotic sequence generated by the piece-wise linear skew tent map can yield a pseudo-random sequence with a desired probability distribution and, consequently, a good ambiguity function. From the above analysis, however, we have seen that the ambiguity function is not as good as expected for the first values of $k$ because the distribution of the chaotic sequence has not yet converged to the permanent and desired distribution, i.e. the uniform distribution in $[-1,1]$.

To circumvent this problem we here consider a down-sampled version of the chaotic sequence, i.e. instead of sequences $\left\{x_{p}, 0 \leq p \leq N_{c}-1\right\}$ defined as in section 4 we consider sequences $\left\{x_{p}^{s}, 0 \leq p \leq N_{c}-1\right\}$ defined by: $x_{p}^{s}=x_{p N_{s}}, 0 \leq$ $p \leq N_{c}-1$ where $N_{s}$ is the sampling rate. Noting that $x_{p+k}^{s}-x_{p}^{s}=x_{N_{s} p+N_{s} k}-$ $x_{N_{s} p}$ the probability density of $x_{p+k}^{s}-x_{p}^{s}$ is the same as the one of $x_{q+N_{s} k}-x_{q}$. For example, the distributions of $x_{p+k}^{s}-x_{p}^{s}$ for $k=1,2, N_{s}=5$ and when $x_{p}$ is generated by 33 with $\mu=0.1$ are plotted in Figure 18 .

Comparing these distributions to the ones in Figures 11 and 12 and for the same values of $k=1,2$ we can see that we achieved the desired uniform probability density for all values of $k$. To analyse the impact of this down-sampling on 

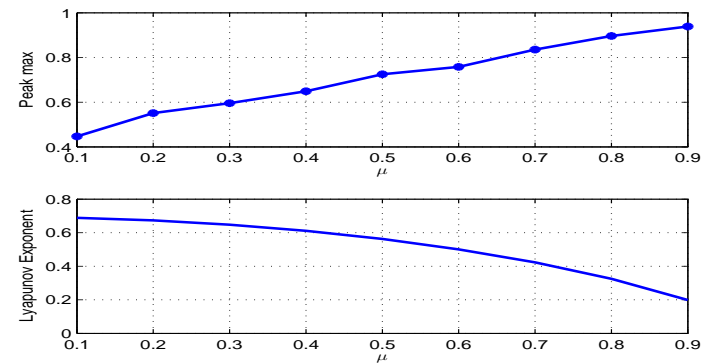

Figure 17: Maximal peak of the ambiguity function $(|R(0, \nu)|$ removed) and Lyapunov exponent versus the bifurcation parameter $\mu$
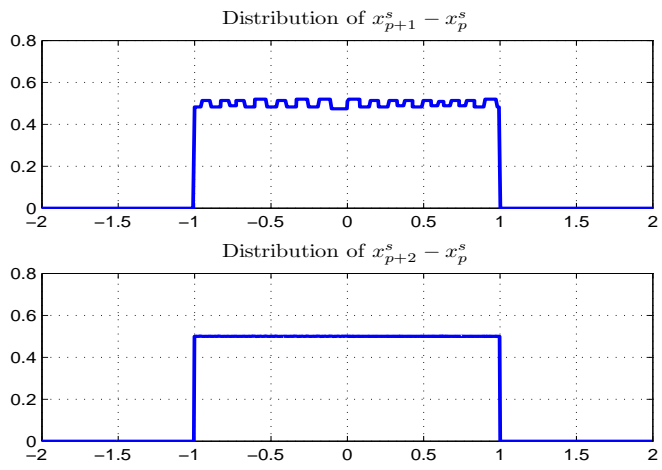

Figure 18: Distribution of $x_{p+1}^{s}-x_{p}$ and $x_{p+2}^{s}-x_{p}$ for $N_{s}=5$

the ambiguity function we plotted on Figure $19|R(k, \nu)|, k=1,2,3$ for $x_{p}$ and

${ }_{320} x_{p}^{S}$. We can clearly see the superiority of the down-sampled sequence $x_{p}^{S}$ with respect to the sequence $x_{p}$.

The resulting ambiguity function in the case $\mu=0.1$ and for $N_{s}=5$ from which we have removed the maximum peaks of $|R(0, \nu)|$ is shown in Figure 20 By comparing this ambiguity function to that of Figure 15 corresponding to ${ }_{325} \mu=0.1$ and for $N_{s}=1$ we can see the disappearance of the high peaks close to $(k, \nu)=(1,0)$ (corresponding to the first side lobe). We also see that the maximum value of the side lobes is reduced while their average value is almost the same. 

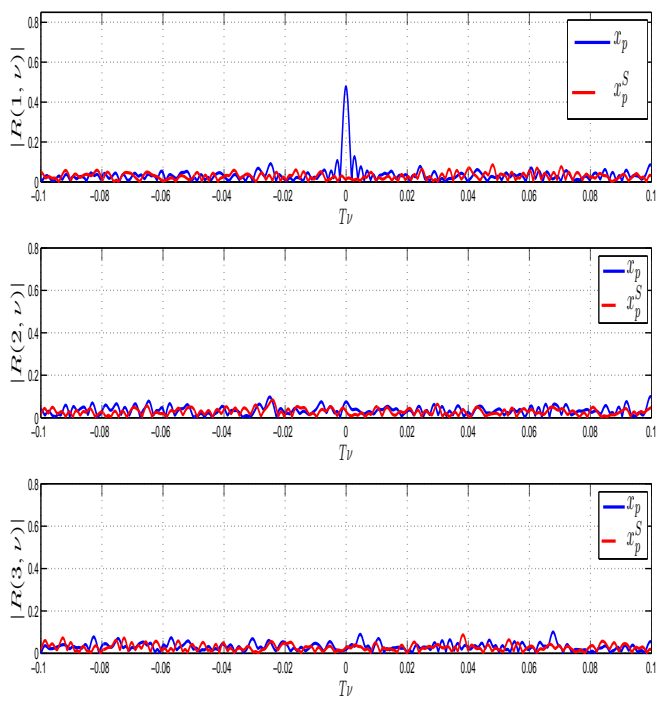

Figure 19: $|R(k, \nu)|, k=1,2,3$ for $x_{p}$ and $x_{p}^{S}$

We have compared the performances of the waveforms presented here to those proposed in [26]. In this work, the authors generated phase coded waveforms from the so-called cyclic-new algorithm (CAN), an iterative and cyclic algorithm minimizing a cost function based on the ambiguity function. By comparing Figures 20 and 21 and in particular the maximum and average values of the side lobes of the ambiguity functions, it can be seen that the sequences based on the chaos proposed with a down sampling give almost the same performances as CAN sequences. The advantage of chaotic waveforms over CAN waveforms is due to the simplicity of their generation and this regardless of their length or number. Indeed by randomly choosing the initial condition in $(33)$ we obtain a new sequence having the same statistical properties as the others. This also gives the possibility of obtaining sequences with desired spectral characteristics. On the contrary, in the case of CAN waveforms, if we want to add a new waveform and / or a longer waveform to those already generated by CAN, further optimization of the cost function is necessary. 


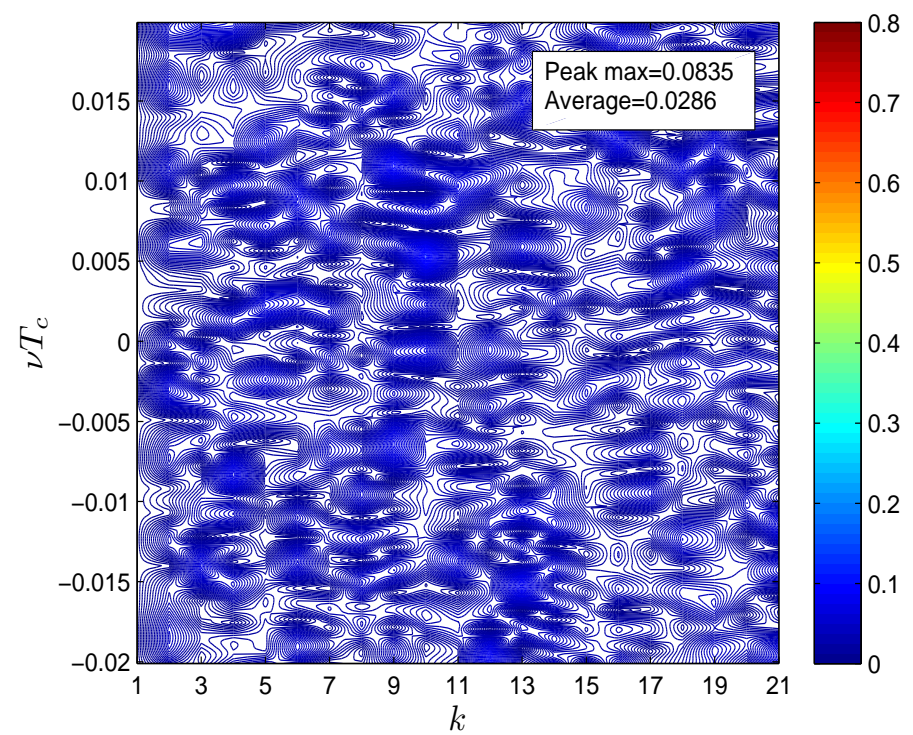

Figure 20: Ambiguity function $(|R(0, \nu)|$ removed) obtained with down-sampled chaotic waveform $(\mu=0.1)$ 


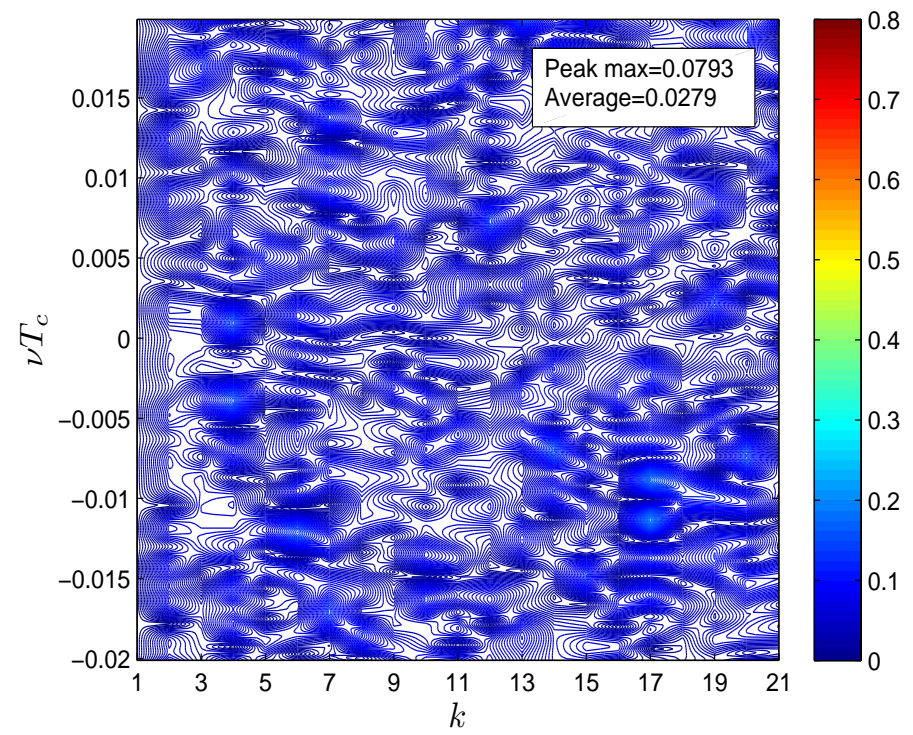

Figure 21: Ambiguity function $(|R(0, \nu)|$ removed) obtained with CAN waveforms

345

\section{Conclusion}

In this paper we adopted a statistical approach to look for sequences that optimize the ambiguity function of a radar system. We have shown that desired sequences can be generated by a random variable with an appropriate distribution and that it is possible to obtain such sequences by generating them using they can be generated of any length and in large numbers. Although generated from a deterministic map with few parameters, they can be considered pseudorandom which makes it possible to study their statistical characteristics. Note that other chaotic sequences could be used and studied.

355 In this paper, we considered the ambiguity function in the case of a basic radar system. It would be easy to demonstrate that the proposed analysis is still valid for the single input-multi-output (SIMO) radar system. The multi-inputmulti-output (MIMO) case is however less straightforward since it involves the 
generation of multiple sequences with good properties of the cross-correlations which are embedded in other parameters such that the transmission antenna geometry. Because of the promising results we have here obtained, we will examine the MIMO case in a future work and adopt the same statistical approach to optimize the ambiguity function by considering the cross-correlation functions of the sequences.

\section{References}

[1] M. A. Richards, Fundamentals of radar signal processing, second edition, IET, Mac Graw-Hill Education (2014).

[2] P. M. Woodward, Probability and information theory, with applications to radar, D. W. Fry W. Higinbotham (1953).

370 [3] D. Eustice, C. Baylis, R. J. Marks, Woodward's ambiguity function: From foundations to applications, Texas Symposium on Wireless and Mi-

口 crowave Circuits and Systems (WMCS) (2015). doi:10.1109/WMCaS. 2015.7233208

[4] M. R. Bell, Information theory and radar waveform design, IEEE Transac375 tions on Information Theory 39 (5) (1993) 1578-1597. doi:10.1109/18. 259642

[5] K. Alhujaili, V. Monga, M. Rangaswamy, Quartic gradient descent for tractable radar slow-time ambiguity function (staf) shaping, IEEE Transactions on Aerospace and Electronic Systems (2019). doi:10.1109/TAES. 2019.2934336

[6] H. E. Najafabadi, H. Leung, P. W. Moo, Unimodular waveform design with desired ambiguity function for cognitive radar, IEEE Transactions

I on Aerospace and Electronic Systems (2019). doi:10.1109/TAES.2019. 2942411 
[7] J. Li, P. Stoica, Mimo radar signal processing, John Wiley and Sons Inc. New Jersey (2009).

[8] H. Sun, F. Brigui, M. Lesturgie, Analysis and comparison of mimo radar waveforms, International Radar Conference, Lille, France (13-17 October 2014). doi:10.1109/RADAR .2014.7060251.

[9] M. Bolhasani, E. Mehrshahi, S. Ghorashi, M. Alijani, Constant envelope waveform design to increase range resolution and sinr in correlated mimo radar, Signal Processing 163 (2019) 59-65. doi:https://doi.org/10. 1016/j.sigpro.2019.05.009

[10] P. Stoica, H. He, J. Li, On designing sequences with impulse-like periodic 395 _ correlation, IEEE Signal Processing Letters 16 (8) (2009) 703-706. doi: 10.1109/LSP. 2009.2021378

[11] H. He, P. Stoica, J. Li, Designing unimodular sequence sets with good correlations - including an application to mimo radar, IEEE Trans. on signal processing 57 (11) (2009) 4391-4405. doi:10.1109/TSP.2009.2025108.

[12] F. Arlery, R. Kassab, U. Tan, F. Lehmann, Efficient gradient method for locally optimizing the periodic/aperiodic ambiguity function, IEEE Radar

口 Conference (RadarConf), Philadelphia, PA, USA (2-6 May 2016). doi: 10.1109/RADAR.2016.7485309.

[13] U. Tan, al, Phase code optimization for coherent mimo radar via a gradient descent, IEEE Radar Conference (RadarConf), Philadelphia, PA, USA (2-6 May 2016). doi:10.1109/RADAR.2016.7485178.

[14] R. Vignesh, G. A. S. Sundarama, K. P. Somana, Design of less-detectable radar waveforms using stepped frequency modulation and coding, Procedia q Computer Science 143 (2018). doi:https://doi.org/10.1016/j.procs. 2018.10 .349 
[15] A. Aubry, A. D. Maio, B. Jiang, S. Zhang, Ambiguity function shaping for cognitive radar via complex quartic optimization, IEEE Trans. on signal processing 61 (22) (2013) 5603-5619. doi:10.1109/TSP.2013.2273885.

[16] G. Cui, Y. Fu, X. Yu, J. Li, Local ambiguity function shaping via unimodular sequence design, IEEE Signal Processing Letters 24 (7) (2017) 977-981. doi:10.1109/LSP.2017.2700396.

[17] J. Yang, G. Cui, X. Yu, Y. Xiao, L. Kong, Cognitive local ambiguity function shaping with spectral coexistence, IEEE Access 6 (2018) 50077-50086. doi:10.1109/ACCESS.2018.2868884.

[18] M. Khanfouci, S. Marcos, Plm sequences for the performance optimization of linear multiuser detectors, Eusipco, Antalya, Turkey (4-8 September 2005).

[19] S. Merhrzi, S. Marcos, S. Belghith, A family of spatiotemporal chaotic sequences outperforming gold ones in asynchronous ds-cdma systems, Eusipco, Florence, Italy (4-8 September 2006).

[20] Z. B. Jemaa, S. Belghith, Chaotic sequences with good correlation properties for mimo radar application, SoftCom Conference, Split, Croatia, (4-8 September 2016). doi:10.1109/SOFTCOM.2016.7772127.

[21] M. S. Willsey, K. M. Cuomo, A. V. Oppenheim, Quasi-orthogonal wideband radar waveforms based on chaotic systems, IEEE Transactions on

口 Aerospace and Electronic Systems 47 (3) (2011) 1974-1984. doi:10.1109/ TAES.2011.5937277.

[22] Y. Jin, H. Wang, W. Jiang, Z. Zhuang, Complementary-based chaotic phase-coded waveforms design for mimo radar, IET Radar, Sonar and Navigation 7 (4) (2013) 371-382. doi:10.1049/iet-rsn.2012.0123.

[23] P. Beckmann, Statistical distribution of the amplitude and the phase of a multiply scattered field, Journal of Research of the National Buureau of Standards-Radio Propagation 66D (3) (1962) 231-240. 
[24] M. Eisencraft, D.M.Kato, L.H.A.Monteiro, Spectral properties of chaotic signals generated by the skew tent map, Signal Processing 90 (1) (2010) 385-390. doi:https://doi.org/10.1016/j.sigpro.2009.06.018

[25] Z. B. Jemaa, D. Fournier-Prunaret, S. Belghith, Kendall's tau based correlation analysis of chaotic sequences generated by piecewise linear maps, International Journal of Bifurcation and Chaos 25 (13) (2015) 385-390. doi:DOI:10.1142/S0218127415501771.

[26] H. He, Waveform design for active sensing systems - a compytational aproach, PhD thesis,University of Florida (2015).

\section{Appendix}

Proof of 24) and 25)

In this appendix we show (24) where we assumed $a=2 l, l$ is a positive integer; the demonstration of 25 is almost the same. For simplicity we begin by the notations: $W=y_{p} \in[-a, a]$ and $Z=\tilde{y}_{p} \in[-1,1]$. It is obvious that if $W=Z+2 k$ where $k$ is an integer, we have $e^{j \pi W}=e^{j \pi Z}$.

We have

455

$$
\begin{gathered}
P[Z<z]=0 ; \forall z \leq-1 \\
P[Z<z]=1 ; \forall z \geq 1
\end{gathered}
$$

For $z \in[-1,2 l-a]$

$$
P[Z<z]=P[-1 \leq Z<z]=\sum_{k=1-l}^{l} P[-1+2 k \leq W<z+2 k]
$$

Knowing that $W$ is uniformly distributed in $[-a, a]$ we have $P[-1+2 k<W<$ $z+2 k]=\frac{z+1}{2 a} ;$ we obtain

$$
P[Z<z]=2 l \frac{z+1}{2 a}=\frac{l}{a}(z+1)
$$

For $z \in[2 l-a, 0]$

$P[Z<z]=P[-1 \leq Z<2 l-a]+P[2 l-a<Z<z]=\frac{l}{a}(2 l-a+1)+\sum_{k=-l}^{l} P[2 l-a+2 k<W<z+2 k]$ 
460

We obtain

$$
P[Z<z]=\frac{l}{a}(2 l-a+1)+\frac{2 l+1}{2 a}(z+a-2 l)
$$

For $z \in[0, a-2 l]$

$$
P[Z<z]=P[-1 \leq Z<0]+P[0<Z<z]
$$

From 47 the first term of this summation is $\frac{1}{2}$, thus

$$
P[Z<z]=\frac{1}{2}+\sum_{k=-l}^{l} P[2 k<W<z+2 k]
$$

We obtain

$$
P[Z<z]=\frac{1}{2}+\frac{2 l+1}{2 a} z
$$

For $z \in[a-2 l, 1]$

$$
P[Z<z]=P[-1<Z<a-2 l]+P[a-2 l<Z<z]
$$

465 From 48 the first term of this summation is $\frac{(2 l+1)(a-2 l)}{2 a}$, thus

$$
P[Z<z]=\frac{(2 l+1)(a-2 l)}{2 a}+\sum_{k=-l}^{l-1} P[a+2 k-2 l<W<z+2 k]
$$

We obtain

$$
P[Z<z]=\frac{(2 l+1)(a-2 l)}{2 a}+\frac{l}{a}(z-a+2 l)
$$

The distribution of $Z$ is obtained by deriving the expressions 4549 and thus we obtain (24).

Proof of 27 and 28

As the demonstrations of the expressions 27 and $(28)$ are similar we show here only 27 .

Let $W=y_{p} \in[-a, a]$ and $Z=\tilde{y}_{p} \in[-1,1]$. It is obvious that if $W=Z+2 k$ where $k$ is an integer, we have $e^{j \pi W}=e^{j \pi Z}$.

Also we have (44) and (45).

${ }_{475}$ For $z \in[-1,2 l-a]$

$$
P[Z<z]=P[-1 \leq Z<z]=\sum_{k=1-l}^{l} P[-1+2 k \leq W<z+2 k]
$$




$$
f_{W}(w)=\left\{\begin{array}{c}
\frac{w+a}{a^{2}}, \forall w \in[-a, 0] \\
\frac{-w+a}{a^{2}}, \forall w \in[0, a]
\end{array}\right.
$$

Thus we have

$$
P[-1+2 k<W<z+2 k]=\left\{\begin{array}{c}
\int_{-1+2 k}^{z+2 k} \frac{-w+a}{a^{2}} d w, \text { if } k \geq 1 \\
\int_{-1+2 k}^{z+2 k} \frac{w+a}{a^{2}} d w, \text { if } k \leq 0
\end{array}\right.
$$

which gives

$$
P[Z<z]=\frac{1}{a^{2}} \sum_{k=1-l}^{0}\left[\frac{1}{2} z^{2}+(a+2 k) z+C_{1}\right]+\frac{1}{a^{2}} \sum_{k=1}^{l}\left[-\frac{1}{2} z^{2}+(a-2 k) z+C_{2}\right]
$$

where $C_{1}$ and $C_{2}$ are constants; By simplifying we obtain

$$
P[Z<z]=\frac{1}{a^{2}}\left(2 a l-l^{2}\right) z+C_{1}+C_{2}
$$

$D=C_{3}+C_{4}$ Thus,

$$
P[Z<z]=\frac{1}{a^{2}}\left[\frac{1}{2} z^{2}+((2 l+1) a-2 l(l+1)) z\right]+C+D
$$

By deriving (50) and (51) we find the distribution of $Z$ for $z<0$, for the symmetry of the problem we deduce the distribution of $Z$ for $z>0$; thus we find

$$
f_{Z}(z)=\left\{\begin{array}{c}
\frac{2 a l-l^{2}}{a^{2}}, \quad \text { if } a-2 l \leq|z| \geq 1 \\
\frac{(2 l+1) a-2 l(l+1)-|z|}{a^{2}}, \text { if } 0<|z| \leq a-2 l
\end{array}\right.
$$

